\title{
Recognition of Process Disturbances for an SPC/EPC Stochastic System Using Support Vector Machine and Artificial Neural Network Approaches
}

\author{
Yuehjen E. Shao \\ Department of Statistics and Information Science, Fu Jen Catholic University, New Taipei City 24205, Taiwan \\ Correspondence should be addressed to Yuehjen E. Shao; stat1003@mail.fju.edu.tw
}

Received 9 March 2014; Accepted 19 May 2014; Published 9 June 2014

Academic Editor: Suohai Fan

Copyright (c) 2014 Yuehjen E. Shao. This is an open access article distributed under the Creative Commons Attribution License, which permits unrestricted use, distribution, and reproduction in any medium, provided the original work is properly cited.

\begin{abstract}
Because of the excellent performance on monitoring and controlling an autocorrelated process, the integration of statistical process control (SPC) and engineering process control (EPC) has drawn considerable attention in recent years. Both theoretical and empirical findings have suggested that the integration of SPC and EPC can be an effective way to improve the quality of a process, especially when the underlying process is autocorrelated. However, because EPC compensates for the effects of underlying disturbances, the disturbance patterns are embedded and hard to be recognized. Effective recognition of disturbance patterns is a very important issue for process improvement since disturbance patterns would be associated with certain assignable causes which affect the process. In practical situations, after compensating by EPC, the underlying disturbance patterns could be of any mixture types which are totally different from the original patterns. This study proposes the integration of support vector machine (SVM) and artificial neural network (ANN) approaches to recognize the disturbance patterns of the underlying disturbances. Experimental results revealed that the proposed schemes are able to effectively recognize various disturbance patterns of an SPC/EPC system.
\end{abstract}

\section{Introduction}

Due to the fact that the process quality is mainly based on the detection and control of the disturbance, it is customized to develop various kinds of monitoring and controlling techniques in the past decade. Statistical process control (SPC) chart is one of the most commonly used techniques to monitor and improve the quality of a process. Typically, a process is considered in an out-of-control state when a plotted point falls outside the control limits or when the SPC charts exhibit nonrandom or systematic patterns [1].

The main function of SPC charts is to monitor and detect the presence of process disturbances when they intrude in the process. A limitation of using traditional SPC charts is that they should monitor the independent process outputs [2-5]. If correlation among process outputs exists, the type I errors would be increased and the detecting capability of SPC charts is seriously decreased. However, the correlation does exist in some continuous and chemical processes [6-10]. One of the effective ways to deal with the difficulty in monitoring correlated outputs for SPC applications is to use the engineering process control (EPC) [11-19]. The use of a suitable EPC action would produce independent process outputs, and the problems of charting correlated outputs by SPC charts can be overcome.

Although EPC provides advantages in helping SPC applications, little work has been reported on addressing the deficiencies of integration of SPC/EPC. The use of EPC may be embedded in the effects of underlying disturbances. That is, the process disturbance patterns are concealed. It causes the identification of the disturbance patterns to become much more difficult $[4,17]$. Effective recognition of those nonrandom patterns is very important because those patterns are usually associated with some specific process disturbances which antagonistically influence the process [20-22]. For example, a step-change disturbance may be resulted from the introduction of new raw materials, workers, or machines or a change in the skill of the operators. A trend disturbance pattern may be associated with a gradual wearing out or deterioration of a tool. Accordingly, the identification of disturbance 


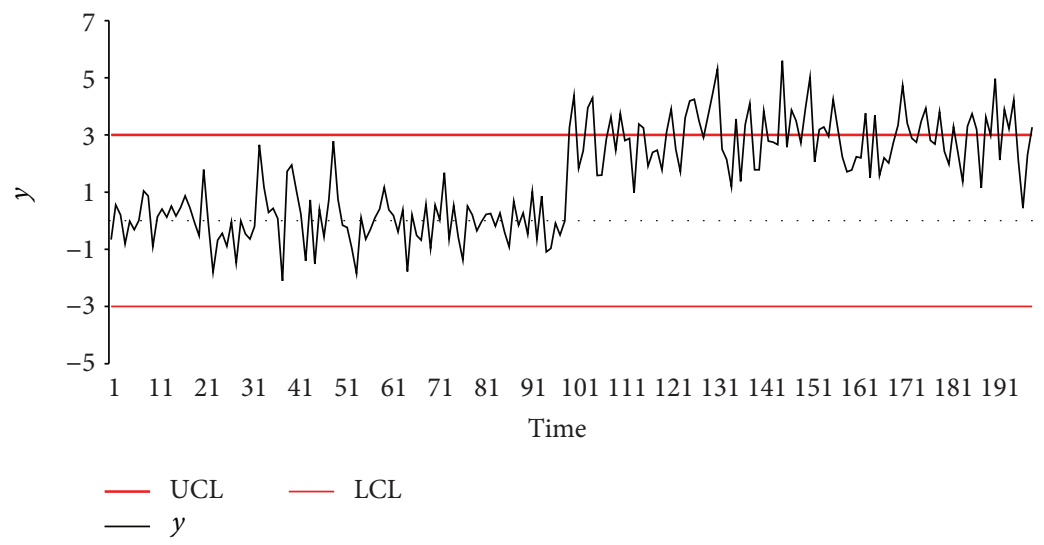

FIGURE 1: The process outputs with the presence of a shift disturbance.

patterns for a combined SPC and EPC (i.e., SPC/EPC) process has become a promising research issue.

Due to their greater generalization ability and superior performance in practical applications [23-29], this study uses artificial neural network (ANN) and support vector machine (SVM) to serve as the classifiers to identify three commonly observed disturbance patterns [20, 30, 31]. Those three process disturbance patterns include shift, trend, and cycle patterns. In this study, we propose the combination of SPC, EPC, and ANN (i.e., the SEA approach) as well as SPC, EPC, and SVM (i.e., the SES approach) to overcome the problems in an SPC/EPC system. Using the proposed mechanisms, this study is able to effectively identify the underlying process disturbance patterns in a correlated process. The superiority of the proposed approaches is addressed with the use of simulated experiments.

The structure of this study is organized as follows. Section 2 describes the difficulty of SPC/EPC system for monitoring a correlated process. The concept of an EPC is introduced in this section as well. Section 3 discusses the proposed approaches for identifying the disturbance patterns for a SPC/EPC system. Section 4 presents a series of simulated experiments which are used to report the performance of the proposed approaches. The final section reports the research findings and concludes this study.

\section{Process Models and the Problem Statements}

The process model without autocorrelation is presented in Section 2.1. An SPC chart is demonstrated to effectively detect a disturbance. Section 2.2 introduces the models of an autocorrelated process and EPC action. The difficulty of recognition of underlying process disturbance patterns is reported. Section 2.3 addresses three types of disturbance models.

2.1. Process Model without Autocorrelation. Suppose that a process, which has no correlation presented, is modeled as follows:

$$
Y_{t+1}=u+a_{t+1} \text {, }
$$

where $Y_{t+1}$ : output deviation at time $t+1, u$ : process mean level, and $a_{t+1}$ : white noise at time $t+1$. The white noise series are assumed to be normally and independently distributed with mean zero and constant variance $\sigma_{a}^{2}$.

Without loss of generality, this study assumes that $\sigma^{2}=1$. Also, suppose that a shift disturbance (with a shift level of 3 ) has intruded into the process after time 101; Figure 1 displays the results by using a typical SPC chart to monitor such process. Observing Figure 1, one is able to notice that the SPC signal is triggered around time 101 (i.e., the value of $Y_{101}$ falls outside of the upper control limit (UCL) and lower control limit (LCL)). Accordingly, the effectiveness of using an SPC chart can be apparently observed.

2.2. Process Model with Autocorrelation. Autocorrelation commonly exists in real processes, and it decreases the monitoring capability of SPC charts [11, 32-34]. Consider an autocorrelated process which is well represented by the following autoregressive model with order 1 model, AR(1) $[19,31,35-37]$ :

$$
Y_{t+1}=\phi Y_{t}+a_{t+1}
$$

where $\phi$ is the unknown parameter and this study arbitrarily sets $\phi=0.9$.

Figure 2 displays the results by using a typical SPC chart to monitor an autocorrelated process which is represented by (2). One can observe that the false alarm signal has been triggered many times since there are no disturbances in this process. The main reason for such poor monitoring of an SPC chart is just simply that the autocorrelation was involved in this process, and it causes the increase in the false alarm rates. Due to the fact that the monitoring capability would be decreased when SPC is used in an autocorrelated process, the EPC is a good alternative method to overcome this difficulty. Consider the following autocorrelated process with the use of EPC adjustment $[15,34]$ :

$$
Y_{t+1}=q X_{t}+d_{t+1}, \quad d_{t+1}=\frac{a_{t+1}}{(1-\phi B)}
$$




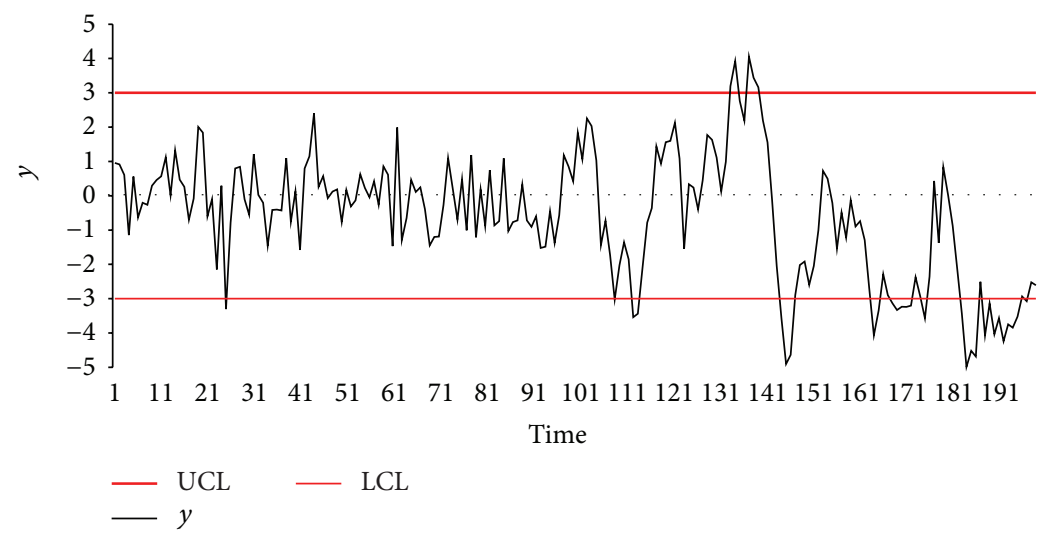

FIgURE 2: The process outputs with the presence of autocorrelation after time 101.

where $X_{t}$ : deviation from the manipulate variable at time $t$, $q$ : process gain, and it is an unknown parameter, $d_{t+1}$ : noise at time $t+1$, and they follow AR(1) model, and $B$ : backward shift operator, and it is defined as $Y_{t} B^{j}=Y_{t-j}$.

Also, the minimum mean squared error (MMSE) control action, one type of EPC, is widely used to tune the process in (3). The MMSE is described as follows [11, 15, 34]:

$$
\widehat{X}_{t}=\phi X_{t-1}-\frac{\phi}{q} a_{t}=\phi X_{t-1}-\frac{\phi}{q} Y_{t} .
$$

Substituting (4) into (3), the following relationship holds:

$$
Y_{t+1}=a_{t+1} .
$$

Equation (4) implies that the process output deviations from the target follow a sequence of independent white noise. Accordingly, the traditional SPC charts can be used to monitor the autocorrelation. Suppose that a disturbance has intruded into the autocorrelated process (i.e., (3)); then the process can be modeled as follows:

$$
Y_{t+1}=q X_{t}+d_{t+1}+D_{t+1}
$$

where $D_{t+1}$ is a certain disturbance at time $t+1$.

2.3. Disturbance Models. The $D_{t+1}$ which is defined in (6) presents a certain disturbance in a process. In this study, we consider three types of disturbance models and they include shift, trend, and cycle disturbances. The corresponding models are described as follows $[35,38]$ :

$$
\begin{aligned}
& \text { Shift: } D_{t}^{S}=D_{t}+a_{t} \\
& \text { Trend: } D_{t}^{T}=t S_{t}+a_{t} \\
& \text { Cycle: } D_{t}^{C}=\sin \left(\frac{2 \pi t}{\psi}\right) U_{t}+a_{t},
\end{aligned}
$$

where $D_{t}^{S}$ : shift disturbance value at time $t, D_{t}$ : level of shift disturbance, and it is assumed that $D_{t}=3$ after shifting, $D_{t}^{T}$ : trend disturbance value at time $t, S_{t}$ : trend slope, and it is assumed to follow a uniform distribution with the range of (0.05-0.1), $D_{t}^{C}$ : cycle disturbance value at time $t, U_{t}$ : cycle amplitude, and it is assumed to follow a uniform distribution with the range of $(1.5-2.5)$, and $\psi$ : cycle period, and it is assumed $\psi=8$.

Figures 1,3 , and 4 show the patterns of those three disturbances. Figures 5, 6, and 7 display the results when the MMSE (i.e., (4)) is used to tune the process with the presence of those three disturbances. Observing Figures 5, 6, and 7, one can notice that the recognition of those disturbance patterns would be a very difficult task. Although there have been many works on systematic pattern recognition [38-42], most of the existing works are concerned with the recognition of the single systematic patterns. As a result, they usually assume that the observed process outputs only possess single basic type of systematic patterns [40-42].

However, in real control chart applications, the observed process data may be mixture of patterns where the original single disturbance pattern was embedded. Compared to the patterns illustrated in Figures 1, 3, and 4, it can be observed in Figures 5 and 6 that the mixture patterns are more difficult to recognize than the single disturbance patterns. Moreover, in practice, mixture process patterns usually result in serious performance degradation for pattern classification [20]. Consequently, how to effectively identify disturbance patterns in the SPC/EPC system is an important and challenging task.

\section{Methodology}

Because of their classification capability, both SVM and ANN have been increasingly used in many areas. Accordingly, this study employs those two techniques to recognize the disturbance patterns in the SPC/EPC systems. In this section, we present the concept of ANN and SVM.

3.1. Support Vector Machine. The basic idea of SVM can be briefly described as follows. SVM initially maps the input vectors into a high dimensional feature space, either linearly or nonlinearly, which is relevant with the selection of the kernel function. The input or feature vectors in the feature space are then classified linearly by a numerically optimized 


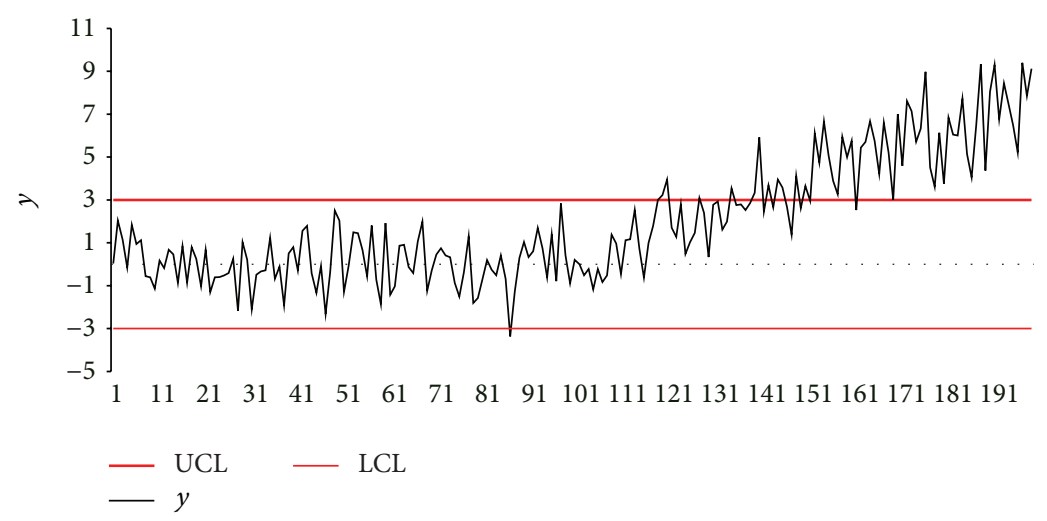

FIGURE 3: The process outputs with the presence of a trend disturbance.

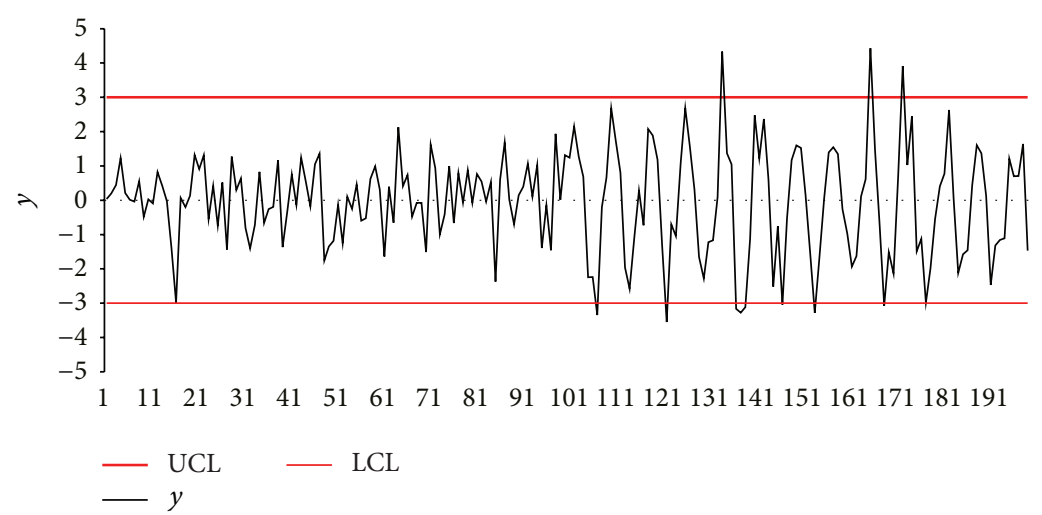

FIGURE 4: The process outputs with the presence of a cycle disturbance.

hyperplane, separating the two classes (this can be extended to multiclass). SVM training always seeks a global optimized solution and the hyperplane depends only on a subset of training examples [43].

Let $\left\{\left(\tilde{x}_{i}, y_{i}\right)\right\}_{i=1}^{n}, \tilde{x}_{i} \in R^{m}, y_{i} \in\{-1,1\}$ be the training set with input vectors $\left(\tilde{x}_{i}\right)$ and outputs $\left(y_{i}\right)$. Here, $n$ is the number of sample observations and $m$ is the dimension of each observation, and $y_{i}$ is known target. The algorithm is to seek the hyperplane $\widetilde{w} \cdot \widetilde{x}_{i}+b=0$, where $\widetilde{w}$ is the vector of hyperplane and $b$ is a bias term, to separate the data from two classes with maximal margin width $2 /\|\widetilde{w}\|^{2}$, and all the points under the boundary are named support vector. In order to optimize the hyperplane, SVM solves the following optimization problem [43]:

$$
\begin{array}{ll}
\text { Min } & \Phi(\widetilde{x})=\frac{1}{2}\|\widetilde{w}\|^{2} \\
\text { s.t. } & y_{i}\left(\widetilde{w}^{T} \tilde{x}_{i}+b\right) \geq 1, \quad i=1,2, \ldots, n
\end{array}
$$

It is difficult to solve (8). Thus, SVM transforms the optimization problem to be dual problem by Lagrange method. The value of $\alpha$ in the Lagrange method must be nonnegative real coefficients. Equation (8) is transformed into the following constrained form [43]:

$$
\begin{array}{ll}
\operatorname{Max} & \Phi(\widetilde{w}, b, \xi, \alpha, \beta)=\sum_{i=1}^{N} \alpha_{i}-\frac{1}{2} \sum_{i=1, j=1}^{N} \alpha_{i} \alpha_{j} y_{i} y_{j} \tilde{x}_{i}^{T} \tilde{x}_{j} \\
\text { s.t. } & \sum_{j=1}^{n} \alpha_{j} y_{j}=0 ; \quad 0 \leq \alpha_{i} \leq C, \quad i=1,2, \ldots, n .
\end{array}
$$

In (9), $C$ is the penalty factor and determines the degree of penalty assigned to an error. It can be viewed as a tuning parameter which can be used to control the trade-off between maximizing the margin and the classification error. In order to separate two classes exactly, add a slack variable $(\xi)$ in the Lagrange equation to have $y_{i}\left(\widetilde{w}^{T} \tilde{x}_{i}+b\right) \geq 1-\xi_{i}, \xi_{i} \geq 0$. An objective of slack variable is to increase the flexible buffer of boundary.

In general, it may not find the linear separate hyperplane for all application data. For problems that cannot be linearly separated in the input space, the SVM uses the kernel method to transform the original input space into a high dimensional feature space where an optimal linear separating hyperplane can be found. The common kernel functions are linear, polynomial, radial basis function (RBF), and sigmoid. 


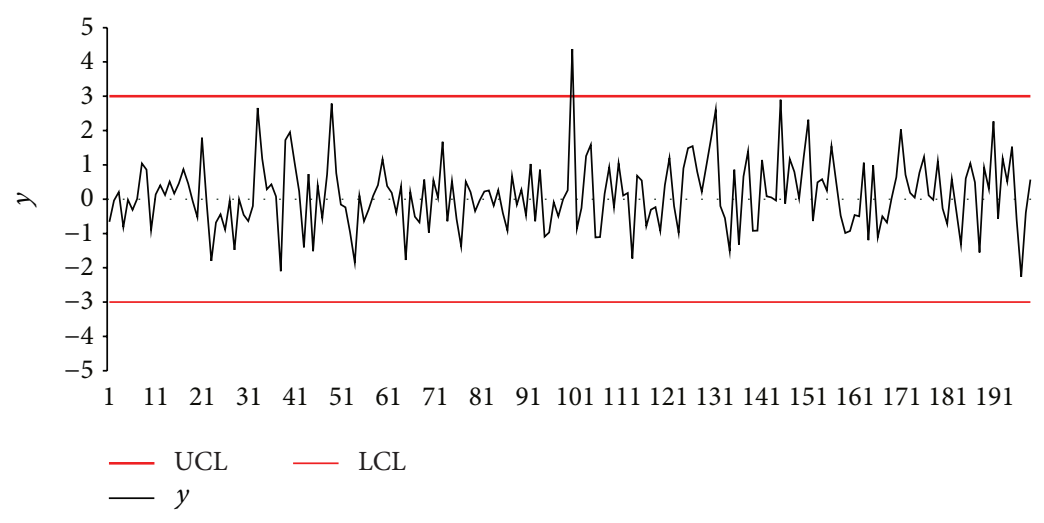

FIGURE 5: The results of using MMSE to tune a process with a shift disturbance.

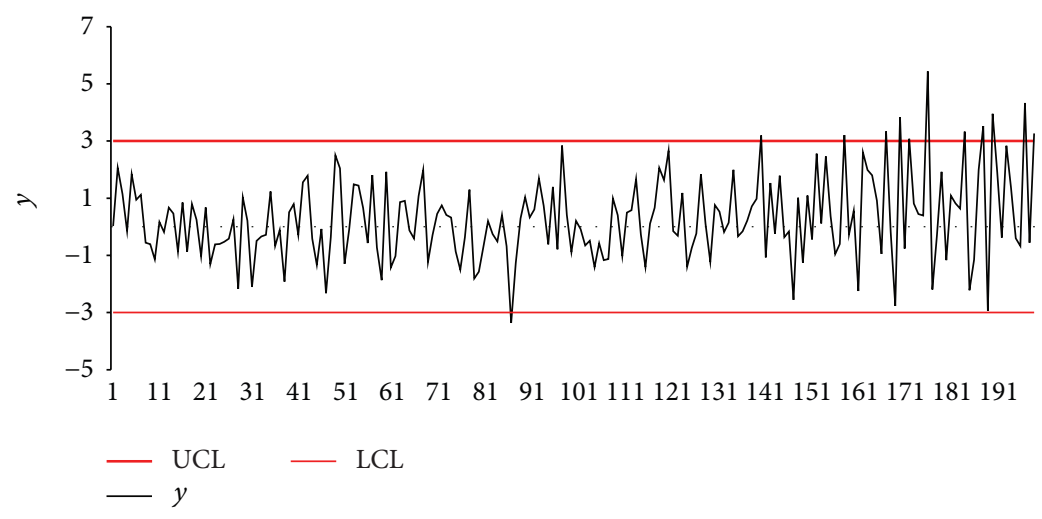

FIGURE 6: The results of using MMSE to tune a process with a trend disturbance.

Although several choices for the kernel function are available, the most widely used kernel function is the RBF which is defined as [44]

$$
K\left(\tilde{x}_{i}, \tilde{x}_{j}\right)=\exp \left(-\gamma\left\|\tilde{x}_{i}-\tilde{x}_{j}\right\|^{2}\right), \quad \gamma \geq 0,
$$

where $\gamma$ denotes the width of the RBF. Consequently, the RBF is employed in this study and the multiclass SVM method is used in this study [45].

3.2. Artificial Neural Network. ANN can be classified into two different categories, feedforward networks and feedback networks [46]. The nodes in the ANN can be divided into three layers: the input layer, the output layer, and one or more hidden layers. The nodes in the input layer receive input signals from an external source and the nodes in the output layer provide the target output signals.

The output of each neuron in the input layer is the same as the input to that neuron. For each neuron $j$ in the hidden layer and neuron $k$ in the output layer, the net inputs are given by

$$
\text { net }_{j}=\sum_{i} w_{j i} \times o_{i}, \quad \operatorname{net}_{k}=\sum_{j} w_{k j} \times o_{j},
$$

where $i(j)$ is a neuron in the previous layer, $o_{i}\left(o_{j}\right)$ is the output of node $i(j)$, and $w_{j i}\left(w_{k j}\right)$ is the connection weight from neuron $i(j)$ to neuron $j(k)$. The neuron outputs are given by

$$
\begin{gathered}
o_{i}=\text { net }_{i}, \\
o_{j}=\frac{1}{1+\exp ^{-\left(\text {net }_{j}+\theta_{j}\right)}}=f_{j}\left(\text { net }_{j}, \theta_{j}\right), \\
o_{k}=\frac{1}{1+\exp ^{-\left(\operatorname{net}_{k}+\theta_{k}\right)}}=f_{k}\left(\text { net }_{k}, \theta_{k}\right),
\end{gathered}
$$

where net ${ }_{j}\left(\right.$ net $\left._{k}\right)$ is the input signal from the external source to the node $j(k)$ in the input layer and $\theta_{j}\left(\theta_{k}\right)$ is the bias. The transformation function shown in (12) is called a sigmoid function. Because this is one of the most commonly utilized functions, it is applied in this study.

The generalized delta rule is the conventional technique used to derive the connection weights of the feedforward network [46]. Initially, a set of random numbers is assigned to the connection weights. Then, to determine the pattern $p$ with a target output vector $t_{p}=\left[t_{p 1}, t_{p 2}, \ldots, t_{p M}\right]^{T}$, the sum of the minimized squared error is given by

$$
E_{p}=\frac{1}{2} \sum_{j=1}^{M}\left(t_{p j}-o_{p j}\right)^{2},
$$




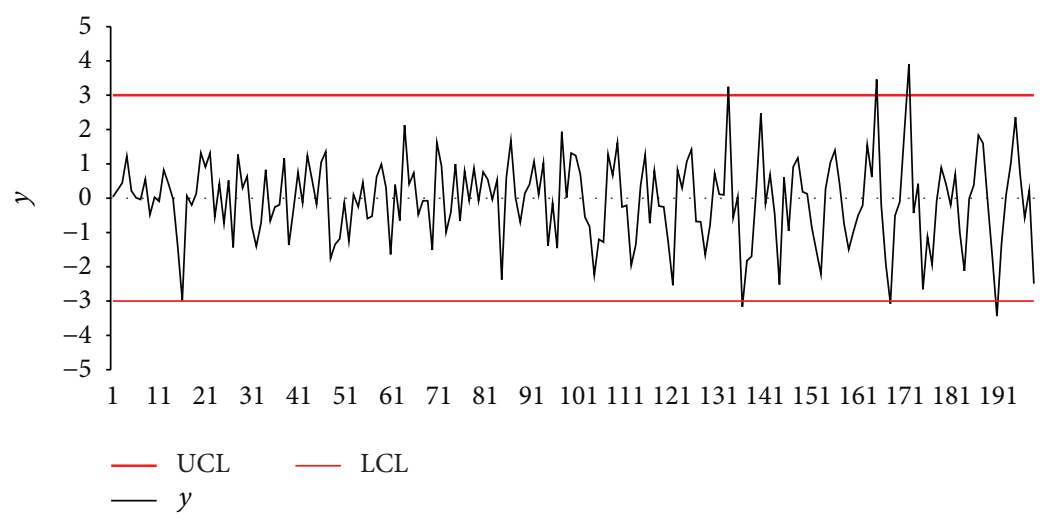

FIGURE 7: The results of using MMSE to tune a process with a cycle disturbance.

TABLE 1: AIR for the proposed models (i.e., recognition of shift and trend disturbances).

\begin{tabular}{lccrr}
\hline Proposed approach & Structures or parameters & Shift & Trend & AIR \\
\hline SES & $C=0.0625 ; \gamma=32$ & 0.986 & 0.826 & $90.60 \%$ \\
\hline & $\{2-2-1\}$ & 0.984 & 0.824 & $90.40 \%$ \\
\multirow{2}{*}{ SEA } & $\{2-3-1\}$ & 0.984 & 0.822 & $90.30 \%$ \\
& $\{2-4-1\}$ & 0.984 & 0.822 & $90.30 \%$ \\
& $\{2-5-1\}$ & 0.988 & 0.822 & $90.50 \%$ \\
\end{tabular}

where $M$ is the number of output nodes. By minimizing the error $E_{p}$ using the technique of gradient descent, the connection weights can be updated by using the following equations:

$$
\Delta w_{j i}(p)=\eta \delta_{p j} o_{p j}+\alpha \Delta w_{j i}(p-1)
$$

where for output nodes

$$
\delta_{p j}=\left(t_{p j}-o_{p j}\right) o_{p j}\left(1-o_{p j}\right),
$$

and for other nodes

$$
\delta_{p j}=\left(\sum_{k} \delta_{p k} * w_{k j}\right) o_{p j}\left(1-o_{p j}\right) .
$$

Note that the learning rate affects the network's generalization and the learning speed to a great extent.

\section{Experimental Results}

This study combines the methodologies of SEA and SES to recognize the types of underlying disturbances for a SPC/EPC system. Suppose a stochastic process is represented in (6) in which $q=0.5, \phi=0.9$, and the variance of the white noise is 1 . This autocorrelated process is fine-tuned with the use of MMSE control action (4). After time 101, a shift, a trend, or a cycle disturbance has intruded in the process.

To use the proposed approaches, this study designs the structure of both SVM and ANN models. In addition, this study considers two cases for recognizing the disturbance patterns. While the first case is the combinations of any two disturbances that would be introduced in the process, the second case is the combination of those three disturbances that would occur in the process. In the case of any two types of disturbances in a process, this study includes 2000 and 1000 data vectors for training and testing stages, respectively. The first 1000 training data vectors are from one of the two disturbances and the remaining 1000 data vectors are from the other disturbances. The testing data structure is the same as the training stage structure. That is, the first 500 data vectors are involved with one disturbance and the remaining 500 data vectors are from the other disturbances. The ratio of $2: 1$ for training and testing data vectors are still applied to the case of three disturbances.

The inputs to SVM and ANN were the MMSE action, $X$, and the process outputs, $Y$. The outputs consist of one node, $Z$. This output node indicates the prediction of the process status. The value of 1,2 , or 3 indicates that the underlying disturbance is shift, trend, or cycle, respectively. For SVM design, since the RBF kernel function is adopted in this study, the performance of SVM is mainly affected by the setting of parameters of two parameters, $C$ and $\gamma$. There are no general rules for the choice of $C$ and $\gamma$. The grid search method uses exponentially growing sequences of $C$ and $\gamma$ to identify good parameters. The parameter set of $C$ and $\gamma$ which generates the highest correct classification rate is considered to be ideal set. In the ANN design, this study defines the term of $\left\{n_{i}-\right.$ $\left.n_{h}-n_{o}\right\}$ as the number of neurons in the input layer, number of neurons in the hidden layer, and number of neurons in 
TABLE 2: AIR for the proposed models (i.e., recognition of shift and cycle disturbances).

\begin{tabular}{lccrr}
\hline Proposed approach & Structures or parameters & Shift & Cycle & AIR \\
\hline SES & $C=0.25 ; \gamma=0.0625$ & 0.842 & 0.770 & $80.60 \%$ \\
\hline & $\{2-2-1\}$ & 0.902 & 0.706 & $80.40 \%$ \\
& $\{2-3-1\}$ & 0.902 & 0.706 & $80.40 \%$ \\
SEA & $\{2-4-1\}$ & 0.904 & 0.710 & 0.710 \\
& $\{2-5-1\}$ & 0.904 & 0.710 & $80.70 \%$ \\
& $\{2-6-1\}$ & 0.902 & $80.60 \%$ \\
\hline
\end{tabular}

TABLE 3: AIR for the proposed models (i.e., recognition of trend and cycle disturbances).

\begin{tabular}{lccrr}
\hline Proposed approach & Structures or parameters & Trend & Cycle & AIR \\
\hline SES & $C=16 ; \gamma=16$ & 0.882 & 0.966 & $92.40 \%$ \\
\hline & $\{2-2-1\}$ & 0.886 & 0.970 & $92.80 \%$ \\
& $\{2-3-1\}$ & 0.860 & 0.996 & $92.80 \%$ \\
SEA & $\{2-4-1\}$ & 0.860 & 0.996 & 0.996 \\
& $\{2-5-1\}$ & 0.860 & 0.996 & $92.80 \%$ \\
& $\{2-6-1\}$ & 0.860 & $92.80 \%$ \\
\hline
\end{tabular}

the output layer, respectively. The hidden nodes in ANN was set to range from $(2 n-2)$ to $(2 n+2)$, where $n$ is the number of input variables.

In the case of recognition for combination of two disturbances, Tables 1, 2, and 3 list the accurate identification rate (AIR) values for the setting of SVM and various setting of ANN topologies. From Table 1, it can be found that both of the $\{2-5-1\}$ and the $\{2-6-1\}$ designs have the highest AIR for the proposed SEA approach. That is, when the shift and/or trend disturbances exist in an SPC/EPC system, the SEA model with either $\{2-5-1\}$ or $\{2-6-1\}$ design could have a $90.50 \%$ chance to accurately recognize the type of underlying disturbance. Also, the proposed SES model with the parameter setting of $C=0.0625$ and $\gamma=32$ could have a $90.60 \%$ chance to accurately recognize the type of underlying disturbance. From Table 2, it can be observed that both SES and SEA approaches provide $80.60 \%$ and $80.70 \%$ chances to accurately recognize the types of shift and/or cycle disturbances in an SPC/EPC system. Table 3 indicates that both SES and SEA approaches provide $92.40 \%$ and $92.80 \%$ chances to accurately recognize the types of shift and/or cycle disturbances in an SPC/EPC system.

In the case of recognition for all three disturbances in an SPC/EPC system, Table 4 displays the AIR for the setting of SVM and various setting of ANN topologies. From Table 4, it can be found that both SES and SEA approaches provide $78.22 \%$ and $77.33 \%$ chances to accurately recognize the types of shift, trend, and cycle disturbances in the SPC/EPC system. Actually, it can be seen that the proposed SES and SEA approaches perform well in recognizing the underlying disturbances. The performance for the proposed approaches does not have significant differences, and the performance can be considered to be satisfactory.

\section{Conclusions}

The use of SPC chart for continuous improvement is an effective strategy for lowering manufacturing costs and improving product value. However, the autocorrelation usually exists in the real manufacturing process, and it causes the increase of false alarm signals of SPC chart. The integration of SPC and EPC has been reported as an alternative to monitor and control the autocorrelated process. Although the EPC compensation may be able to overcome the autocorrelation problems, it causes the underlying disturbance patterns to be embedded. Accordingly, it becomes very difficult to recognize the types of underlying disturbances in an SPC/EPC system.

This study proposes the SES and SEA mechanisms to recognize the types of underlying process disturbances. Three commonly seen disturbances are used in this study for evaluating the performance of the proposed method. Experimental results showed that the proposed schemes are able to produce the satisfactory AIR rate in the tested data sets. According to the experimental results, it can be concluded that the proposed schemes can effectively recognize disturbance patterns in an SPC/EPC system.

In addition to the three types of disturbances which were discussed in this study, there exist other disturbances. For example, the systematic or stratification can also be observed in the process. An attempt to recognize more types of disturbances would be a valuable contribution to this area of research. Also, the issue of recognition of the disturbance patterns for the multivariate systems can be extended as the future research direction [47, 48]. Finally, because more disturbance patterns need to be recognized, the extreme learning machine (ELM) classification scheme is under investigation. 
TABLE 4: AIR for the proposed models (i.e., recognition of all three disturbances).

\begin{tabular}{|c|c|c|c|c|c|}
\hline Proposed approach & Structures or parameters & Shift & Trend & Cycle & AIR \\
\hline SES & $C=8 ; \gamma=16$ & 0.746 & 0.792 & 0.808 & $78.20 \%$ \\
\hline \multirow{5}{*}{ SEA } & $\{2-2-1\}$ & 0.746 & 0.782 & 0.782 & $77.00 \%$ \\
\hline & $\{2-3-1\}$ & 0.740 & 0.786 & 0.786 & $77.07 \%$ \\
\hline & $\{2-4-1\}$ & 0.740 & 0.786 & 0.786 & $77.07 \%$ \\
\hline & $\{2-5-1\}$ & 0.740 & 0.790 & 0.786 & $77.20 \%$ \\
\hline & $\{2-6-1\}$ & 0.728 & 0.784 & 0.808 & $77.33 \%$ \\
\hline
\end{tabular}

\section{Conflict of Interests}

The author declares that there is no conflict of interests regarding the publication of this paper.

\section{Acknowledgments}

This work is partially supported by the National Science Council of Taiwan, Grant no. NSC 102-2221-E-030-019. The author also gratefully acknowledges the helpful comments and suggestions of the reviewers, which have improved the presentation.

\section{References}

[1] W. Hachicha and A. Ghorbel, "A survey of control-chart pattern-recognition literature (1991-2010) based on a new conceptual classification scheme," Computers and Industrial Engineering, vol. 63, no. 1, pp. 204-222, 2012.

[2] D. C. Montgomery and C. M. Mastrangelo, "Some statistical process control for autocorrelation data (with discussion)," Journal of Quality Technology, vol. 23, no. 3, pp. 179-193, 1991.

[3] S. Bisgaard and M. Kulahci, "Quality quandaries: the effect of autocorrelation on statistical process control procedures," Quality Engineering, vol. 17, no. 3, pp. 481-489, 2005.

[4] Y. E. Shao, C.-J. Lu, and C.-C. Chiu, "A fault detection system for an autocorrelated process using SPC/EPC/ANN AND SPC/EPC/SVM schemes," International Journal of Innovative Computing, Information and Control, vol. 7, no. 9, pp. 5417-5428, 2011.

[5] J. G. Requeijo and J. Cordeiro, "Implementation of the statistical process control with autocorrelated data in an automotive manufacturer," International Journal of Industrial and Systems Engineering, vol. 13, no. 3, pp. 325-344, 2013.

[6] B. Wu and J.-B. Yu, "A neural network ensemble model for on-line monitoring of process mean and variance shifts in correlated processes," Expert Systems with Applications, vol. 37, no. 6, pp. 4058-4065, 2010.

[7] C. W. Zobel, D. F. Cook, and Q. J. Nottingham, “An augmented neural network classification approach to detecting mean shifts in correlated manufacturing process parameters," International Journal of Production Research, vol. 42, no. 4, pp. 741-758, 2004.

[8] R.-S. Guh, "Real-time recognition of control chart patterns in autocorrelated processes using a learning vector quantization network-based approach," International Journal of Production Research, vol. 46, no. 14, pp. 3959-3991, 2008.

[9] C. M. Wright, D. E. Booth, and M. Y. Hu, "Joint estimation: SPC method for short-run autocorrelated data," Journal of Quality Technology, vol. 33, no. 3, pp. 365-378, 2001.
[10] D. W. Apley and J. Shi, "The GLRT for statistical process control of autocorrelated processes," IIE Transactions, vol. 31, no. 12, pp. 1123-1134, 1999.

[11] Y. E. Shao, "Integrated application of the cumulative score control chart and engineering process control," Statistica Sinica, vol. 8, no. 1, pp. 239-252, 1998.

[12] G. Box and T. Kramer, "Statistical process monitoring and feedback adjustment-a discussion," Technometrics, vol. 34, no. 3, pp. 251-285, 1992.

[13] Y. E. Shao and C. C. Chih, "Developing identification techniques with the integrated use of SPC/EPC and neural networks," Quality and Reliability Engineering International, vol. 15, no. 4, pp. 287-294, 1999.

[14] W. Jiang and K.-L. Tsui, "SPC monitoring of MMSE- and PIcontrolled processes," Journal of Quality Technology, vol. 34, no. 4, pp. 384-398, 2002.

[15] Y. E. Shao, G. C. Runger, J. Haddock, and W. A. Wallace, "Adaptive controllers to integrate SPC and EPC," Communications in Statistics B: Simulation and Computation, vol. 28, no. 1, pp. 1336, 1999.

[16] F. Tsung and K.-L. Tsui, "A mean-shift pattern study on integration of SPC and APC for process monitoring," IIE Transactions, vol. 35, no. 3, pp. 231-242, 2003.

[17] C.-C. Chiu, Y. E. Shao, T.-S. Lee, and K.-M. Lee, "Identification of process disturbance using SPC/EPC and neural networks," Journal of Intelligent Manufacturing, vol. 14, no. 3-4, pp. 379388, 2003.

[18] C.-J. Lu, C.-M. Wu, C.-J. Keng, and C.-C. Chiu, "Integrated application of SPC/EPC/ICA and neural networks," International Journal of Production Research, vol. 46, no. 4, pp. 873-893, 2008.

[19] G. Box and S. Narasimhan, "Rethinking statistics for quality control," Quality Engineering, vol. 22, no. 2, pp. 60-72, 2010.

[20] C.-J. Lu, Y. E. Shao, and P.-H. Li, "Mixture control chart patterns recognition using independent component analysis and support vector machine," Neurocomputing, vol. 74, no. 11, pp. 1908-1914, 2011.

[21] Y. E. Shao, C.-J. Lu, and Y.-C. Wang, "A hybrid ICA-SVM approach for determining the quality variables at fault in a multivariate process," Mathematical Problems in Engineering, vol. 2012, Article ID 284910, 12 pages, 2012.

[22] C. J. Lu, Y. E. Shao, and C. C. Li, "Recognition of concurrent control chart patterns by integrating ICA and SVM," Applied Mathematics \& Information Sciences, vol. 8, no. 2, pp. 681-689, 2014.

[23] Y. E. Shao and C.-D. Hou, "Change point determination for a multivariate process using a two-stage hybrid scheme," Applied Soft Computing Journal, vol. 13, no. 3, pp. 1520-1527, 2013. 
[24] Y. E. Shao and C.-D. Hou, "Fault identification in industrial processes using an integrated approach of neural network and analysis of variance," Mathematical Problems in Engineering, vol. 2013, Article ID 516760, 7 pages, 2013.

[25] Y. E. Shao, C.-J. Lu, and C.-D. Hou, "Hybrid soft computing schemes for the prediction of import demand of crude oil in Taiwan," Mathematical Problems in Engineering, vol. 2014, Article ID 257947, 11 pages, 2014.

[26] Y. E. Shao, "Body fat percentage prediction using intelligent hybrid approaches," The Scientific World Journal, vol. 2014, Article ID 383910, 8 pages, 2014.

[27] Y. E. Shao, C. D. Hou, and C. C. Chiu, "Hybrid intelligent modeling schemes for heart disease classification," Applied Soft Computing, vol. 14, pp. 47-52, 2014.

[28] Y. E. Shao and C. D. Hou, "Hybrid artificial neural networks modeling for faults identification of a stochastic multivariate process," Abstract and Applied Analysis, vol. 2013, Article ID 386757, 10 pages, 2013.

[29] Y. E. Shao, "Prediction of currency volume issued in taiwan using a hybrid artificial neural network and multiple regression approach," Mathematical Problems in Engineering, vol. 2013, Article ID 676742, 9 pages, 2013.

[30] W. Dai, Y. E. Shao, and C.-J. Lu, "Incorporating feature selection method into support vector regression for stock index forecasting," Neural Computing and Applications, vol. 23, no. 6, pp. 15511561, 2013.

[31] S.-Y. Lin, R.-S. Guh, and Y.-R. Shiue, "Effective recognition of control chart patterns in autocorrelated data using a support vector machine based approach," Computers and Industrial Engineering, vol. 61, no. 4, pp. 1123-1134, 2011.

[32] C. M. Mastrangelo and D. C. Montgomery, "SPC with correlated observations for the chemical and process industries," Quality and Reliability Engineering International, vol. 11, no. 2, pp. 7989, 1995.

[33] F. W. Faltin, C. M. Mastrangelo, G. C. Runger, and T. P. Ryan, "Considerations in the monitoring of autocorrelated and independent data," Journal of Quality Technology, vol. 29, no. 2, pp. 131-133, 1997.

[34] J. F. MacGregor, T. J. Harris, and J. D. Wright, "Duality between the control of processes subject to randomly occurring deterministic disturbances and ARIMA stochastic disturbances," Technometrics, vol. 26, no. 4, pp. 389-397, 1984.

[35] J. Arkat, S. T. A. Niaki, and B. Abbasi, "Artificial neural networks in applying MCUSUM residuals charts for $A R(1)$ processes," Applied Mathematics and Computation, vol. 189, no. 2, pp. 18891901, 2007.

[36] A. D. Karaoglan and G. M. Bayhan, "A regression control chart for autocorrelated processes," International Journal of Industrial and Systems Engineering, vol. 16, no. 2, pp. 238-256, 2014.

[37] A. D. Karaoglan, "An integrated neural network structure for recognizing autocorrelated and trending processes," Mathematical and Computational Applications, vol. 16, no. 2, pp. 514-523, 2011.

[38] S. K. Gauri and S. Chakraborty, "Feature-based recognition of control chart patterns," Computers and Industrial Engineering, vol. 51, no. 4, pp. 726-742, 2006.

[39] R.-S. Guh and J. D. T. Tannock, "Recognition of control chart concurrent patterns using a neural network approach," International Journal of Production Research, vol. 37, no. 8, pp. 1743-1765, 1999.
[40] S. K. Gauri and S. Chakraborty, "Recognition of control chart patterns using improved selection of features," Computers and Industrial Engineering, vol. 56, no. 4, pp. 1577-1588, 2009.

[41] R.-S. Guh and Y.-R. Shiue, "On-line identification of control chart patterns using self-organizing approaches," International Journal of Production Research, vol. 43, no. 6, pp. 1225-1254, 2005.

[42] C.-H. Wang and W. Kuo, "Identification of control chart patterns using wavelet filtering and robust fuzzy clustering," Journal of Intelligent Manufacturing, vol. 18, no. 3, pp. 343-350, 2007.

[43] V. N. Vapnik, The Nature of Statistical Learning Theory, Springer, Berlin, Germany, 2000.

[44] V. Cherkassky and Y. Ma, "Practical selection of SVM parameters and noise estimation for SVM regression," Neural Networks, vol. 17, no. 1, pp. 113-126, 2004.

[45] C.-W. Hsu and C.-J. Lin, "A comparison of methods for multiclass support vector machines," IEEE Transactions on Neural Networks, vol. 13, no. 2, pp. 415-425, 2002.

[46] B. Cheng and D. M. Titterington, "Neural networks: a review from a statistical perspective," Statistical Science, vol. 9, no. 1, pp. 2-30, 1994.

[47] S. T. A. Niaki and M. Davoodi, "Designing a multivariate-multistage quality control system using artificial neural networks," International Journal of Production Research, vol. 47, no. 1, pp. 251-271, 2009.

[48] H. B. Hwarng and Y. Wang, "Shift detection and source identification in multivariate autocorrelated processes," International Journal of Production Research, vol. 48, no. 3, pp. 835-859, 2010. 


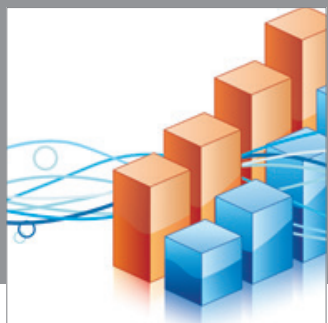

Advances in

Operations Research

mansans

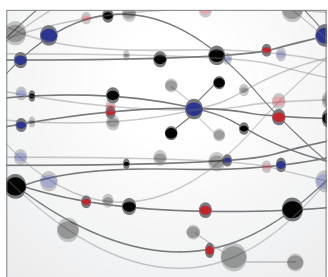

The Scientific World Journal
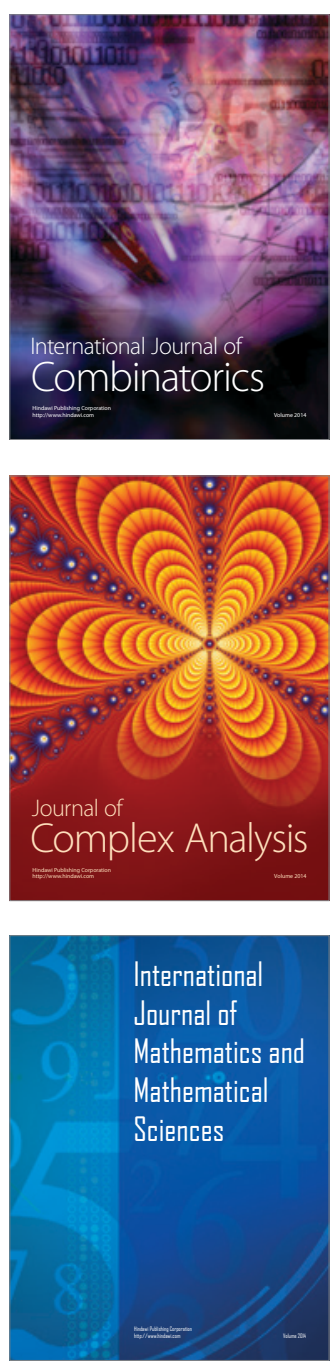
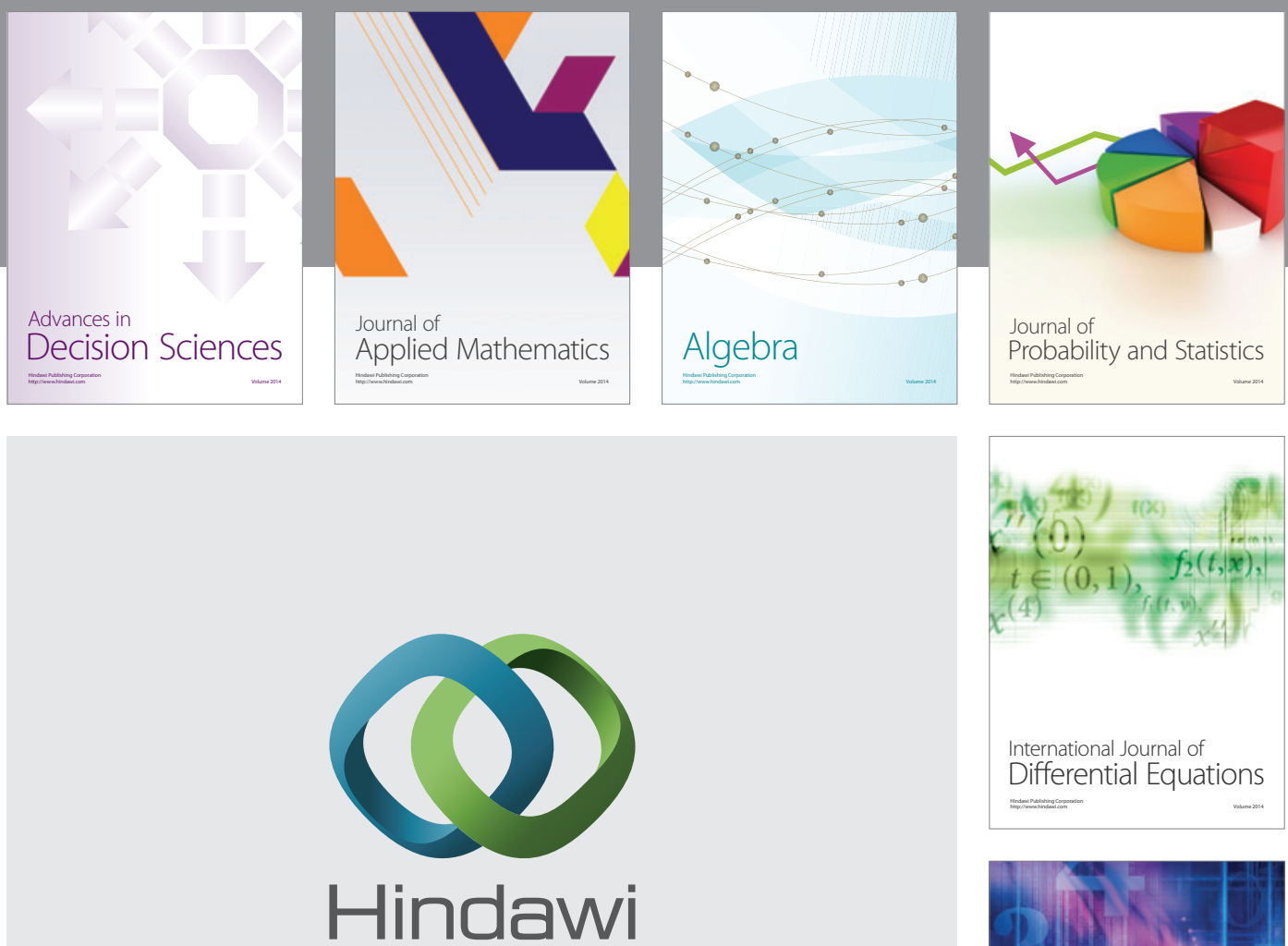

Submit your manuscripts at http://www.hindawi.com
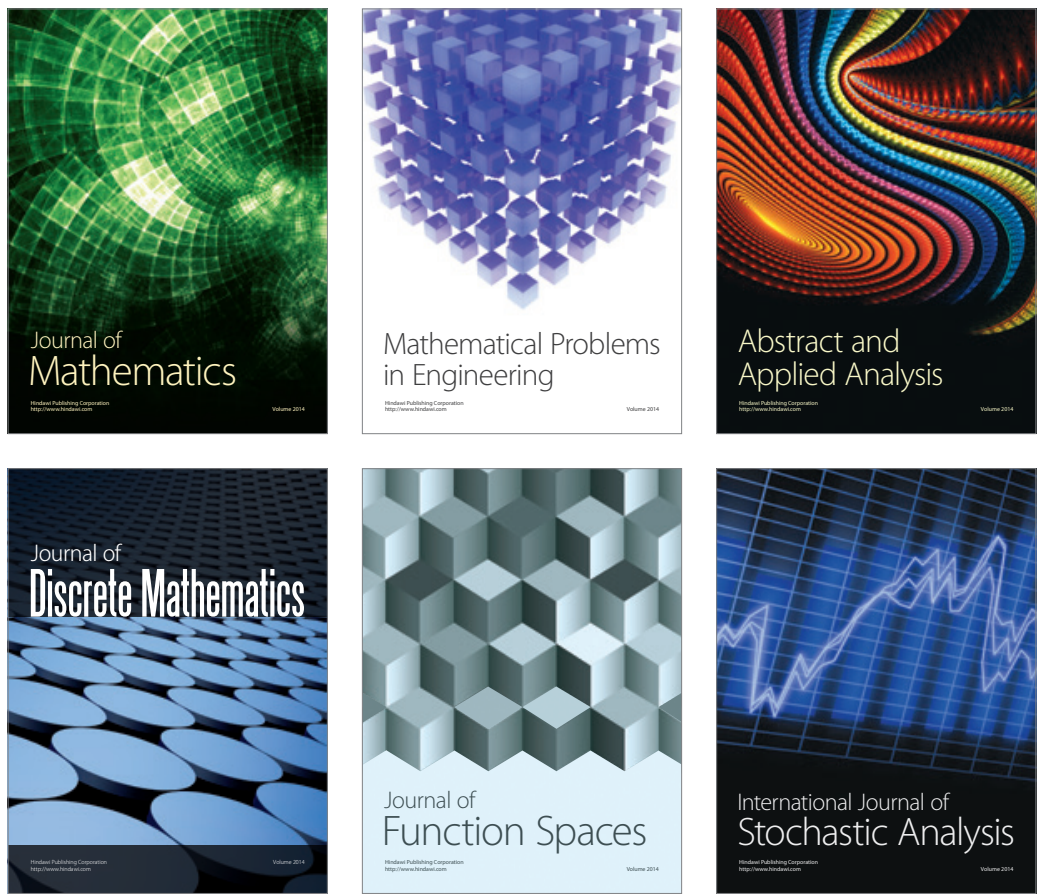

Journal of

Function Spaces

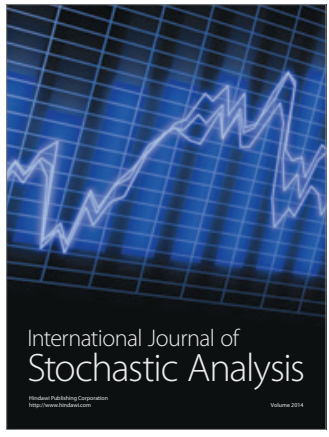

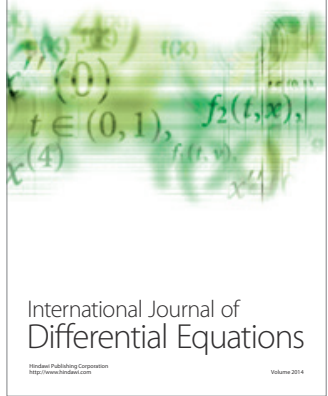
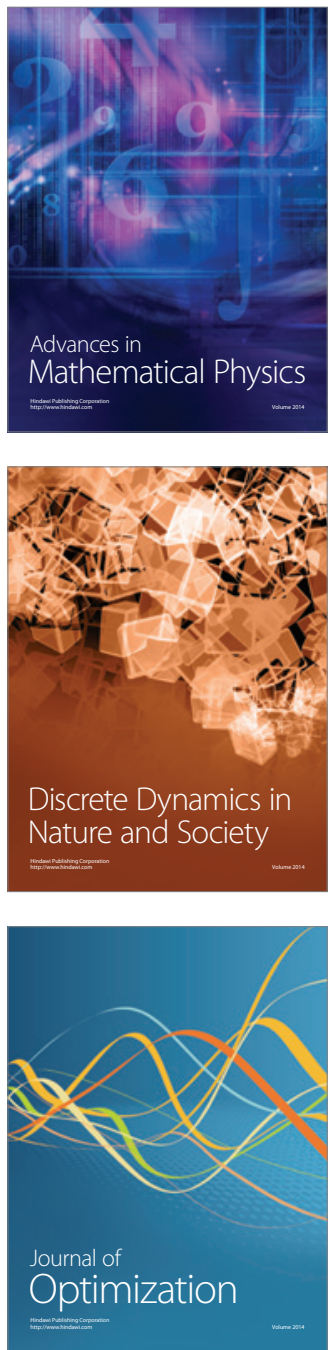